\title{
Gasto de hogares durante la hospitalización de menores derechohabientes, con diagnóstico de leucemia, en dos hospitales en México
}

Arnoldo Rocha-García, M en C, (1) Patricia Hernández-Peña, MSP, Dra en Econ, ${ }^{(2)}$ Silvia Ruiz-Velazco, D ra, en Mat, (3) Leticia Avila-Burgos, M en C, ${ }^{(4)}$ Teresa Marín-Palomares, MC, ${ }^{(5)}$ Eduardo Lazcano-Ponce, M en C, Dr en C. ${ }^{(6)}$

Rocha-García A, Hernández-Peña P, Ruiz-Velazco S, Avila-Burgos L, Marín-PalomaresT, Lazcano-Ponce E. Gasto de hogares durante la hospitalización de menores derechohabientes, con diagnóstico de leucemia, en dos hospitales en México. Salud Publica Mex 2003;45:285-292.

El texto completo en inglés de este artículo está disponible en: http://www.insp.mx/salud/index.html

\section{Resumen}

Objetivo. Estimar el gasto de los hogares durante la primera hospitalización en 51 menores de 15 años de edad con leucemia, atendidos en dos hospitales del Instituto Mexicano del Seguro Social, en México durante 1997. Ma terial y métodos. Estudio transversal hecho en 1997 en el Distrito Federal y en León, G uanajuato. Se aplicó un cuestionario a los padres de 51 menores de 15 años de edad con diagnóstico de leucemia, hospitalizados por primera vez, en dos unidades del Instituto Mexicano del Seguro Social. Se capturó la información de los costos directos e indirectos enfrentados por los hogares durante esa primera hospitalización. Se aplicó el Indice de Precios al Consumidor (1997-2002) para expresar las estimaciones en precios de 2002. Se estimaron indicadores de gasto promedio y gastos catastróficos. Se establecieron los factores asociados, mediante un modelo de regresión lineal, utilizando el gasto total durante la hospitalización como variable dependiente. Resultados El costo promedio por paciente hospitalizado es de 7318 pesos. El 86\% corresponde a gastos asociados con la atención y $14 \%$ a costos indirectos. Para $14 \%$ de los hogares este gasto fue catastrófico. En $47 \%$ de los casos la
Rocha-García A, Hernández-Peña P, Ruiz-Velazco S, Avila-Burgos L, Marín-PalomaresT, Lazcano-Ponce E. Out-of-pocket expenditures during hospitalization of young leukemia patients with state medical insurance in two Mexican hospitals. Salud Publica Mex 2003;45:285-292.

The English version of this paper is available at: http://www.insp.mx/salud/index.html
A bstract
Objective. To estimate out-of-pocket expenditures for health care during the first hospitalization of children treat- ed for leukemia in two hospitals of the Mexican Institute of Social Security (Instituto Mexicano del Seguro Social - IMSS-). Material and Methods A cross-sectional study was conducted in Mexico City and Leon, Guanajato, Me- xico in 1997.The study population consisted of the parents of 51 children under 15 years of age diagnosed with leuke- mia, who were hospitalized for the first time in two IMSS hospitals. A questionnaire was applied to participants to obtain direct and indirect expenditures during that period. Consumer price indexes (1997-2002) were used to esti- mate expenditure prices for 2002.Average expenditures and catastrophic expenditures were estimated. Factors associat- ed with expenditures were analyzed using a linear regres- sion model in which the dependent variable was the total household expenditures during hospitalization. Results The average household cost per hospitalization was 7318 pe- sos, $86 \%$ of which corresponded to medical care and $14 \%$ to indirect costs. Catastrophic expenditures occurred in $14 \%$ of households. In $47 \%$ of household expenditures

(1) Instituto Mexicano del Seguro Social (IMSS). León, Guanajuato, México.

(2) O rganización Mundial de la Salud. Ginebra, Suiza.

(3) Instituto de Investigaciones en Matemáticas A plicadas y Superiores. Universidad N acional Autónoma de México (UN AM). México, DF, México.

(4) Centro de Investigación en Sistemas de Salud. Instituto N acional de Salud Pública (IN SP). Cuernavaca, Morelos, México.

(5) Hospital General Centro Médico N acional La Raza. IMSS. México, DF, México.

(6) Dirección de Enfermedades Crónicas en el Centro de Investigaciones en Salud Poblaciona, IN SP. Cuernavaca, Morelos, México.

Fecha de recibido: 30 de noviembre de 2001 • Fecha de aprobado: 10 de marzo de 2003

Solicitud de sobretiros: D r. Eduardo Lazcano Ponce. Director de Enfermedades Crónicas. Centro de Investigaciones en Salud Poblacional. Instituto N acional de Salud Pública. Avenida U niversidad 655, Colonia Santa María A huacatitlán. Cuernavaca, Morelos, México.

Correo electrónico: elazcano@ correo.insp.mx 
erogación rebasó $100 \%$ de su ingreso disponible durante el perio do. Estos gastos se asociaron con lugar de residencia, nivel de ingreso y tipo de seguro. Conclusiones. Ser derechohabiente de la seguridad social reduce los gastos de bolsillo por atención directa de los pacientes, pero no reduce los gastos complementarios, que pueden resultar onerosos para una elevada proporción de hogares. El costo de la primera hospitalización significó, en más de la mitad de los casos estudiados, el consumo de los ahorros, el endeudamiento o la venta de propiedades de los hogares, y dificultó la continuidad del tratamiento. El texto completo en inglés de este artículo está disponible en: http://www.insp.mx/salud/index.html

Palabras clave: gasto de hogares; gastos catastróficos; leucemia; costos de la atención en salud; ho spitalización; México exceeded $100 \%$ of the total household income during the hospitalization period. Expenditures during hospitalization were associated with place of residence, income level, and type of medical insurance. Conclusions Being an IMSS policyholder decreased out-of-pocket expenditures, but not complementary expenditures, which may still be unaffordable for a large segment of the population. For more than a half of the households studied, continuity of care was compromised, as expenditures during the first hospitalization entailed using up savings, going into debt, and/or selling household property. The English version of this paper is available at: http://www.insp.mx/salud/index.html

Key words:out-of-pocket expenditure; catastrophic expenditure; leukemia; health care costs; hospitalization; Mexico
E 1 gasto en salud de los hogares incluye el pago de bienes y servicios para la prevención, detección, tratamiento y rehabilitación, es decir, los componentes que constituyen el costo directo de la atención. ${ }^{* 1-3}$ En los hogares con acceso a servicios de la seguridad social la mayor parte de este costo se efectúa mediante un prepago, a través de contribuciones del empleador, el trabajador y las transferencias del gobierno. ${ }^{5}$ La seguridad social usualmente no cubre los gastos complementarios de los hogares, los que además enfrentan los costos indirectos de la atención. Los gastos en traslado, estancia y alimentación son mayores cuando la atención del enfermo se lleva a cabo en un lugar diferente al de residencia. El Instituto Mexicano del Seguro Social (IMSS) cubre parcialmente los gastos de transporte al compensar el costo de un traslado del paciente con un acompañante a la unidad de atención, cuando ésta se ubica fuera del lugar de residencia; ${ }^{5}$ esta compensación no se incrementa cuando se requiere que el paciente sea hospitalizado y visitado por sus familiares, ni se realiza en forma automática, sino bajo gestión específica del derechohabiente. 5,6

\footnotetext{
* El costo económico de una enfermedad tiene principalmente dos componentes: los costos directos y los indirectos. ${ }^{1-3}$ Los primeros incluyen el valor de los recursos necesarios para ejecutar las actividades de prevención, atención y rehabilitación de los problemas de salud. ${ }^{1}$

Los costos indirectos consideran el valor del producto perdido debido a la enfermedad o muerte prematura ${ }^{4}$ e incluyen tres catego rías: ${ }^{2}$ a) los costos de tiempo del paciente y su familia relacionados con el tratamiento y atención; en este rubro se incluyen los tiempos de traslado y espera; b) pérdidas del ingreso, debido al daño que la enfermedad ocasiona en la capacidad para trabajar; esto considera el ausentismo laboral por enfermedad, o cuidado del enfermo, y c) pérdida en la producción, debido a la muerte prematura.
}

En México los estudios de gasto de los hogares han estimado que el de bolsillo representa aproximadamente $49 \%$ del gasto total en salud. ${ }^{7}$ Se calcula que entre 2 y 3 millones de hogares destinan una tercera parte de su ingreso para la atención de su salud, y que este porcentaje tiende a ser mayor en los hogares más pobres. ${ }^{7}$ Según la Encuesta Nacional de Salud (ENSAII) de 1994,8 en hogares con acceso a la seguridad social los gastos por traslado equivalen a la mitad de un salario mínimo, y por medicamentos, a 1.5 salarios mínimos. ${ }^{8}$ La magnitud de estos gastos es importante si se considera que el ingreso per cápita de los asegurados en el IMSS oscila entre uno y tres salarios mínimos. ${ }^{6}$ Además de estos desembolsos, el ingreso esperado del paciente y de los familiares que lo cuidan ${ }^{2,4}$ se ve afectado, debido a la pérdida del ingreso ocasionado por el ausentismo laboral. La monetarización de las pérdidas de ingreso asociadas con la enfermedad es escasa en México, pero estimaciones preliminares calculan que puede representar hasta $28 \%$ del costo económico de la enfermedad. ${ }^{3}$ Los estudios hechos en países con un alto nivel de ingreso per cápita reportan un porcentaje mayor, hasta $52 \%$, del costo total de la enfermedad. ${ }^{1}$

Los gastos catastróficos constituyen un indicador de la inequidad financiera del sistema. Sin embargo, su medición hasta ahora es escasa. Por otra parte, los estudios de gastos en salud de los hogares generalmente estiman casi de manera exclusiva los gastos asociados con los costos directos, excluyendo los gastos complementarios. ${ }^{9}$

El impacto económico de la atención de la salud, al aunar todos los gastos asociados con la atención y la pérdida del ingreso, puede resultar muy elevado respecto de los ingresos de los hogares, y constituir una barrera económica ${ }^{7}$ que obstaculice la búsqueda, acep- 
tación y continuidad de la atención, lo que resulta determinante en términos de oportunidad y manejo de los pacientes. Este documento presenta la estimación de los gastos de bolsillo que realizan los hogares derechohabientes del IMSS, durante la primera hospitalización de pacientes menores de 15 años de edad, con diagnóstico de leucemia, así como los factores asociados con dichos gastos. Se estiman también los costos derivados de la pérdida del ingreso por ausencia laboral de los padres. Los resultados se discuten a la luz de la magnitud de estos gastos con relación al ingreso del hogar y con el impacto potencial en la viabilidad y continuidad del tratamiento de estos pacientes. La leucemia es un padecimiento que representa, actualmente, la primera causa de muerte por cáncer en edad pediátrica, y cuya atención requiere usualmente de varios internamientos y de un control médico continuo. ${ }^{10}$

\section{Material y métodos}

El diseño del estudio es transversal. La muestra se integró con todos los pacientes menores de 15 años de edad, diagnosticados por primera vez con leucemia y que fueron trasladados para su tratamiento inicial en cualquiera de los dos hospitales pediátricos de tercer nivel de atención del IMSS, durante el segundo semestre de 1997. Se incluyó un total de 52 pacientes, obteniendo una tasa de respuesta de $98 \%$.

El estudio estimó el gasto de bolsillo de los hogares durante el periodo de la hospitalización de los pacientes, cuantificando la parte del costo directo cubierta por los hogares, y los principales costos indirectos enfrentados por las familias. La información se captó mediante un cuestionario con preguntas abiertas y cerradas. En 98\% de los casos se entrevistó a los padres al darse de alta al paciente, ya fuera por remisión o por fallecimiento. Se recogió la información sobre escolaridad de los padres, edad de éstos y del paciente, lugar de residencia (se consideraron foráneos cuando vivían a más de 30 minutos de traslado de los hospitales donde se les proporcionó atención), ocupación del jefe de familia, ingreso mensual del hogar, gastos habituales en alimentos y el tipo de esquema de seguridad al que pertenecían: régimen ordinario, facultativo o voluntario.

Se preguntó a los padres el monto y tipo de gastos asociados directamente con la primera atención hospitalaria de su hijo(a). Las respuestas se refirieron, principalmente, a los gastos por la compra de medicamentos, material de curación, transporte, gastos de estancia y alimentos, y compra de artículos de higiene o aseo personal. Se identificaron, además, en un grupo reducido de hogares, pagos a personal para ayudar a las tareas domésticas o cuidado de los otros hijos cuando la ausencia de los padres en el hogar, especialmente de la madre, hizo necesaria la contratación de este tipo de servicios. Finalmente, se preguntó a los padres el ingreso perdido por ausencia en su trabajo durante el evento.

El costo total para los hogares durante la primera hospitalización se integró con la suma de los gastos y las pérdidas económicas asociadas con la primera hospitalización. Debido a que las estimaciones se hicieron en $1997 \mathrm{y}$ han ocurrido cambios de valor monetario desde entonces, se actualizaron los pesos para 2002 considerando el Indice Nacional de Precios al Consumidor ${ }^{11}$ del periodo 1997-2002, y mediante la siguiente fórmula: ${ }^{12}$

Gastos $_{2002}=$ Gasto $_{1997}\left(1+\frac{\mathrm{INPC}_{2002}-\mathrm{INPC}_{1997}}{\mathrm{INPC}_{1997}}\right)$

INPC: Indice Nacional de Precios al Consumidor

Se realizó análisis univariado, utilizando medidas de tendencia central, y bivariado, utilizando comparaciones de medianas. El costo total de la primera hospitalización se comparó con el ingreso anual, así como con el ingreso percibido durante el periodo de la hospitalización. El ingreso disponible se estimó restando al ingreso el gasto rutinario en alimentos. Cuando los gastos de bolsillo medidos excedieron 30\% del ingreso total disponible, se clasificaron como gastos catastróficos. ${ }^{7}$

Se construyó un modelo de regresión lineal utilizando como variable dependiente el gasto total, y como variables independientes la escolaridad del padre, el ingreso por familia, el tipo de seguro (colapsado en dos categorías) y el lugar de residencia. Se evaluó la presencia de colinealidad utilizando para ello el número de condición: cuando éste es mayor de 20 se consideró que existían problemas de colinealidad. ${ }^{13} \mathrm{El}$ análisis se hizó en Stata versión 6, y en SPSS versión 10.

\section{Resultados}

Los enfermos presentaban los tipos de leucemia más frecuentes en el país, a saber, la linfoblástica aguda 38 casos, $74.5 \%$, y la mieloblástica aguda 11 casos, $21.5 \%$. La leucemia granulocítica crónica es la menos frecuente, lo cual coincide con lo reportado en la literatura, y correspondió a dos casos, $4 \%{ }^{10}$

Contra lo que usualmente se reporta en la literatura internacional, la mayoría de los pacientes pertenecía al sexo femenino, $63 \%$; y predominó el grupo 
de menores de cinco años de edad, $52.9 \%$, lo cual coincide con los resultados obtenidos por Berrino. ${ }^{14}$ Más de $40 \%$ de los casos presentaban desnutrición de primer grado.

Con relación al lugar de residencia, $45 \%$ de los pacientes residía en el Estado de México y $90 \%$ provenía de áreas urbanas. El 65\% de los pacientes eran foráneos respecto del sitio donde se ubican los centros de atención.

En $72.6 \%$ de los casos los padres de los pacientes no rebasaban los 40 años de edad. Se trata de familias con un máximo de seis miembros en $66.7 \%$ de los casos, incluyendo a los padres e hijos; asimismo, en $61 \%$ de las familias el paciente era el primogénito o segundo hijo. Predominaron los padres de baja escolaridad: $25 \%$ con primaria incompleta y $28 \%$ con primaria completa. El 49\% de los padres laboraba en el sector industrial y $43 \%$ en el de los servicios (cuadro I). La mayor parte del ingreso de los hogares dependía de la remuneración del padre. Los ingresos mensuales per cápita fueron muy heterogéneos, ya que oscilaron entre $\$ 296.675$ y $\$ 6230$, con un promedio de $\$ 998.514$ pesos en precios de 2002.

El $70.6 \%$ de los hogares, 36 casos, perciben de uno a tres salarios mínimos (cuadro I). Durante el tiempo que permaneció hospitalizado el paciente, $25 \%$ de los hogares percibieron $\$ 3891.33$ o menos, 50\% ganaron entre \$3 954 y \$10 635.8, y 25\% restante tuvieron ingresos superiores a $\$ 10666.2$ pesos en precios de 2002.

La estancia hospitalaria varió entre 4 y 85 días, con un promedio de 42.3 días (cuadro II). Los gastos de los hogares durante este periodo fueron por concepto de transporte, alimentos, medicamentos y material de curación. Todos los hogares realizaron gastos en transporte y casi todos en alimentos; los gastos menos frecuentes correspondieron a medicamentos y material de curación (en seis casos, $11.6 \%$, y cinco casos, 9.8\%, respectivamente). En 34 hogares, 66\%, se reportó la existencia de "otros gastos" (aseo personal, entre otros). Los costos indirectos asociados con el ausentismo laboral y el pago de personal por ayuda en las tareas domésticas se reportaron en 13 casos, 25\% de los hogares.

La figura 1 muestra los componentes analizados en este trabajo. Los rubros que en particular contribuyeron con un mayor porcentaje a la integración del costo total de la primera hospitalización fueron los alimentos (34\%) y el transporte (30\%). Los costos indirectos o de oportunidad de los hogares representaron cerca de $14.2 \%$ (gastos en el hogar por ausencia de los padres, $6.8 \%$, pérdidas de salario por ingresos no percibidos, $7.4 \%$ (cuadro II y figura 1)). El costo total de los hogares representó un promedio de $\$ 7318.01$; si se considera el total de días de hospitalización por pa-

\section{Cuadro I \\ Características SOcioeconómicas de las familias ENTREVISTADAS, DERECHOHABIENTES DEL INSTITUTO Mexicano del Seguro Social. León, Guanajuato y Distrito Federal, México, 1997}

\begin{tabular}{lcl} 
& \multicolumn{2}{c}{ Ocupación del jefe de familia } \\
\cline { 2 - 3 } Total & $\%$ \\
$\begin{array}{l}\text { Sector } \\
\text { Primario (agricultura, } \\
\text { ganadería y pesca) }\end{array}$ & 4 & 7.9 \\
\hline $\begin{array}{l}\text { Secundario (industria y } \\
\text { construcción) }\end{array}$ & 25 & 49 \\
\hline Servicios & 22 & 43.1
\end{tabular}

Tipo de aseguramiento

Tipo de régimen

O rdinario (asegurado

por patrón) $\quad 43 \quad 84.3$

Facultativo (comprado por

$\begin{array}{lll}\text { familiar de trabajador del IMSS) } & 5 & 9.8\end{array}$

Voluntario (comprado

por el público general)

$3 \quad 5.9$

Ingreso por familia* ingreso per cápita ${ }^{\ddagger}$

Salarios mínimos

\begin{tabular}{|c|c|c|c|c|}
\hline$<2$ & 15 & 29.4 & $296.7 \mathrm{a}$ & 4133.5 \\
\hline $2-3$ & 21 & 41.2 & $3135.7 a$ & 4999 \\
\hline$>3$ & 15 & 29.4 & 5001 & 6230.6 \\
\hline
\end{tabular}

* 0 btenido con la suma de ingresos de miembros económicamente activos ₹ Ingreso mensual entre integrantes de la familia expresado en pesos a precios de 2002

ciente el costo que enfrentaron los hogares por día de hospitalización fue de $\$ 287$ pesos en precios de 2002. El gasto de bolsillo representó la mayor parte del costo total medido (92.59\%, \$6 952 pesos) y los ingresos perdidos tan sólo $7.4 \%$.

Para valorar la importancia de los desembolsos durante la primera hospitalización, respecto del ingreso percibido durante ese periodo, debe recordarse que usualmente, en los hogares de bajo ingreso, la mayor parte del gasto familiar se dirige a gastos básicos dentro de una canasta de satisfactores, indispensable para la subsistencia, y la proporción disponible para gastos adicionales suele ser muy reducida. ${ }^{9}$ Cuando se comparan los costos en el hogar con el ingreso disponible durante el periodo de hospitalización, encontramos que únicamente en $10 \%$ de los casos (cinco hogares) este costo fue inferior a 30\% del ingreso; para $43 \%$ de los 


\section{Cuadro II \\ INFORMACIÓN SOBRE LOS GASTOS DE BOLSILLO REALIZADOS DURANTE LA HOSPITALIZACIÓN. Instituto Mexicano del Seguro Social, 1997}

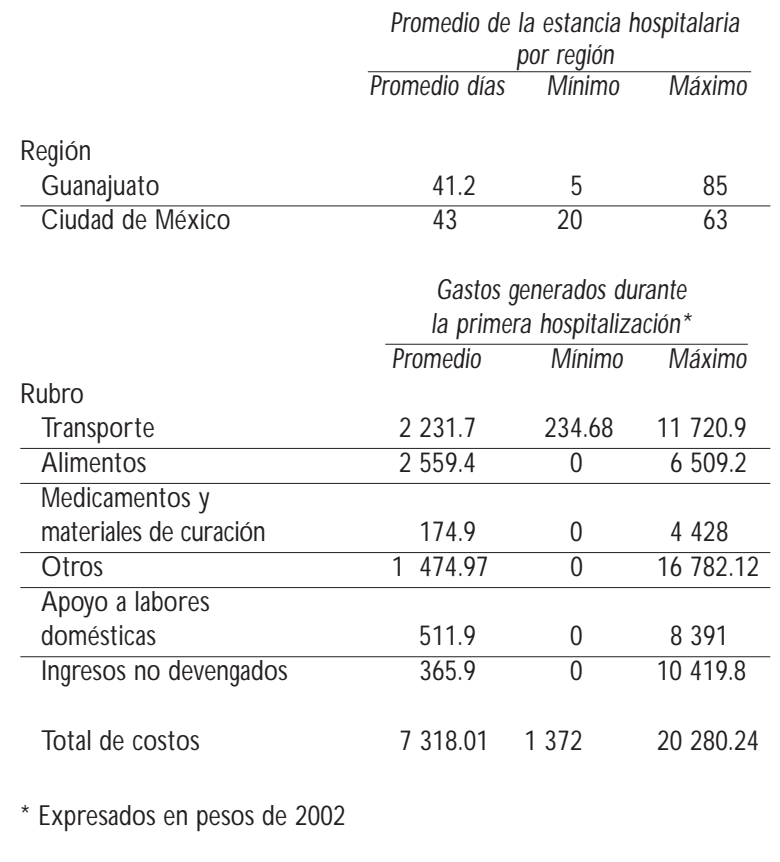

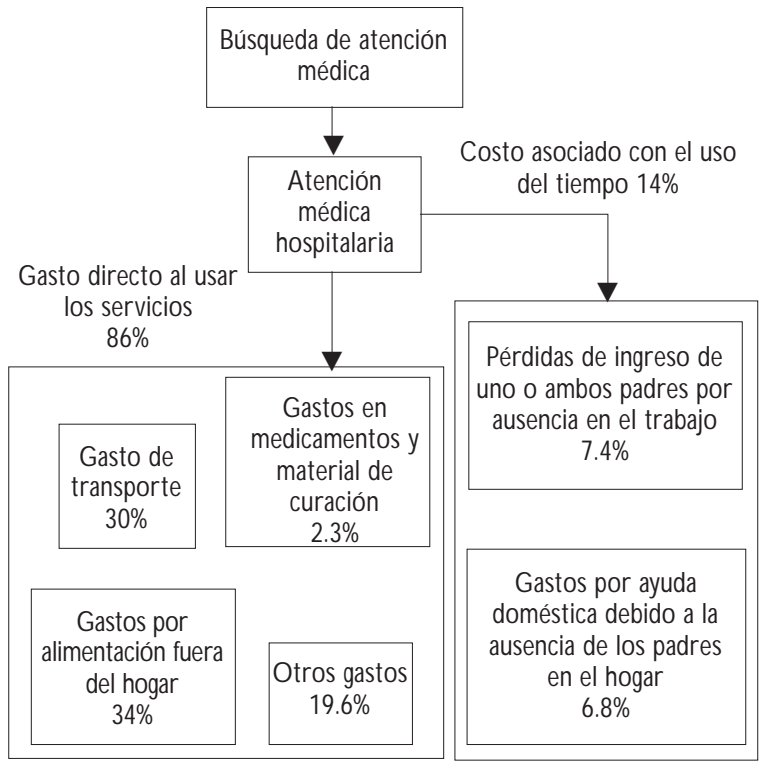

Figura 1. Costos de los hogares por la atención de PACIENTES DE LEUCEMIA DURANTE SU PRIMERA HOSPITALIzación. Instituto Mexicano del Seguro Social, León, Guanajuato y Distrito Federal, México, 1997 hogares representó entre 31 y 100\%, y para el restante $47 \%$ de los hogares dicho costo fue superior a $100 \%$ de su ingreso disponible. El 90\% de los hogares que participaron en el estudio tuvo gastos catastróficos si éstos se comparan con el ingreso obtenido durante el periodo de la primera hospitalización del enfermo (cuadro III).

Los costos que tuvieron los hogares por la primera hospitalización respecto de su ingreso anual disponible fue muy heterogéneo (1-74\%), con una mediana de $22 \%$. Se encontró que para $14 \%$ de los hogares dichos gastos correspondieron a más de $30 \%$ de los ingresos anuales, por lo que se consideran catastróficos.

Es importante señalar que los hogares cuentan con redes de apoyo que, frecuentemente, contribuyen con préstamos y aportaciones directas durante la hospitalización del enfermo. En nuestro estudio, 35\% de los casos informaron haber recibido ayuda monetaria de familiares y amigos, mientras que $7.8 \%$ se vieron obligados a endeudarse para cubrir los gastos de la hospitalización. Una parte contó con apoyo en sus gastos de traslado, ya que el enfermo fue transferido al centro de hospitalización por ambulancias de la propia institución, o bien, recibieron viáticos por una sola ocasión durante la primera hospitalización del hijo. Resultó un hallazgo que quienes tienen derecho a los apoyos semanales para transporte, no los tramitaran en ninguno de los casos.

Se construyó un modelo de regresión lineal que toma como variable dependiente el costo total de los hogares, es decir, la suma del gasto realizado al usar los servicios más el costo asociado con el uso del tiempo. Existe una asociación significativa con el lugar de residencia: cuando los hogares de los pacientes se si-

\section{Cuadro III}

\section{Proporción gasto/Ingreso durante el Periodo de hospitalización. Instituto MeXicano del Seguro Social. León, Guanajuato y Distrito Federal, México, 1997}

\begin{tabular}{lrrrr} 
Porcentaje & Total & \multicolumn{1}{c}{$\begin{array}{c}\text { Mínimo } \\
\%\end{array}$} & $\begin{array}{c}\text { Máximo } \\
\%\end{array}$ \\
$<30$ & 5 & 10 & 12.4 & 26.6 \\
\hline $31-50$ & 4 & 8 & 30.2 & 43.6 \\
\hline $51-100$ & 18 & 35 & 51.7 & 97.5 \\
\hline $101-200$ & 15 & 29 & 100.5 & 193.7 \\
\hline$>200$ & 9 & 18 & 211.3 & 2217.4 \\
Total & 51 & 100 & 12.4 & 2217.4 \\
\hline
\end{tabular}


túan a más de $30 \mathrm{~km}$ de distancia, o a más de 30 minutos de traslado de los centros de tratamiento, el costo fue $42 \%$ más alto que el de aquellos que vivían a menor distancia o a menor tiempo de traslado (IC $95 \%$ de $1.9 \%$ a $107.2 \%)$. Otro factor importante en la determinación del costo total durante la hospitalización fue el tipo de seguro. Los pacientes dentro del régimen de seguro ordinario, es decir, asegurados por un patrón, tuvieron costos $51.6 \%$ menores que quienes adquirieron un seguro facultativo o voluntario (IC 95\% 31.1\% a $85.6 \%$ ). Además, los resultados sugieren que el costo total se asocia positivamente con el ingreso del hogar y la escolaridad del padre, si bien, esta última fue marginalmente significativa (cuadro IV). El 48\% de la variación del costo total de los hogares estuvo explicada por el modelo $\left(r^{2}=0.478\right)$. El número condicional fue de 15.6, por lo que no se encuentran problemas de colinealidad.

\section{Discusión}

El gasto de bolsillo en salud, medido en las encuestas de ingresos y gastos de hogares, suele dirigirse hacia la atención misma, ${ }^{15}$ esto es, al pago del médico, medicamentos y otros insumos médicos. En el caso de la población cubierta por la seguridad social, el prepago por contribuciones mancomunadas periódicas otorga a los hogares acceso a los recursos institucionales dirigidos al tratamiento de los pacientes, incluyendo la hospitalización, ya que la propia institución dispone generalmente de la infraestructura, el personal y los medicamentos necesarios para brindar la atención requerida a la población bajo su responsabilidad. Sin embargo, esto no ocurre en el caso de la atención de padecimientos complejos como la leucemia, donde ciertos gastos en medicamentos y material de curación fueron cubiertos por los hogares. Existen gastos complementarios que no se incluyen en los planes de la seguridad social, o que sólo los cubren parcialmente, por lo que los hogares deben destinar sus propios recursos para facilitar la utilización de los servicios.

Los gastos hechos en el momento de acudir a los servicios de salud, a diferencia de lo ocurrido con la aportación a la seguridad social, por lo general no se encuentran presupuestados dentro del gasto regular de los hogares. Cuando no han sido previstos y cubiertos en forma de prepago éstos se efectúan según la capacidad de compra y la disponibilidad de recursos, y compiten con el consumo de otros bienes y servicios. ${ }^{8}$ Nuestros datos confirman que, en el caso de la población asegurada que estaba hospitalizada por leucemia, si bien el gasto de bolsillo no se dirigió a sufragar la atención debido a que ésta fue cubierta a través de las cuotas (solamente en cinco casos se reportó este tipo de gasto), sí realizó desembolsos importantes para facilitar el acceso a los centros de atención.

En nuestro estudio el gasto en salud de los hogares se elevó con el ingreso; este resultado es similar a lo reportado en la literatura internacional, ${ }^{16,17} \mathrm{y}$ a 10 documentado, en el ámbito nacional, en la Encuesta Nacional de Ingresos y Gastos de Hogares ${ }^{18}$ (ENIGH). Asimismo, los resultados muestran que la magnitud de los gastos puede ser muy elevada respecto del ingreso anual de los hogares. Así, 14\% de los hogares informó haber incurrido en gastos catastróficos en relación con su ingreso anual, proporción más alta que la reportada a escala nacional donde el promedio es de $4.3 \%$, aunque este porcentaje se eleva hasta $20 \%$ en los hogares con menor ingreso y desciende hasta $1 \%$ en los de mayor ingreso. ${ }^{8}$ Las cifras reportadas nacionalmente, sin embargo, cuantifican los gastos catastróficos dirigidos a cubrir los costos directos de la atención, que en esta población representaron un monto menor. En el ámbito nacional existe la posibilidad de que la población con gastos complementarios a la atención, como el transporte, eleve la proporción de grupos afectados

\section{Cuadro IV \\ Factores asociados al gasto total durante la primera hospitalización. Instituto Mexicano del Seguro Social. León, Guanajuato y Distrito Federal, México, 1997}

\begin{tabular}{|c|c|c|c|c|}
\hline Variables & Coeficientes & $t$ & p & IC $95 \%$ \\
\hline & 107.66 & 12.493 & 0.000 & $50.63-\quad 228.9$ \\
\hline Ingreso familiar anual & 0.9998 & -5.029 & 0.000 & $0.9997-\quad 1.0002$ \\
\hline Escolaridad del padre & 1.0410 & 1.720 & 0.092 & $0.9932-\quad 1.0911$ \\
\hline Tipo de seguro (ordinario/facultativo + voluntario) & 0.5164 & -2.634 & 0.012 & $0.3117-\quad 0.8560$ \\
\hline Tipo de localidad (local/foráneo) & 1.4258 & 1.912 & 0.062 & $1.0191-\quad 2.0719$ \\
\hline
\end{tabular}


por gastos catastróficos, o explique la ausencia de atención en grupos que la necesitan.

Como era de esperarse por el tipo de padecimiento, el promedio de días de hospitalización supera con mucho el promedio nacional de seis días por enfermedad, y de casi siete por accidente a escala nacional. ${ }^{8}$ Debido a esto, los hogares deben asignar una proporción importante de su ingreso disponible para su manejo; un elevado grupo de ellos no alcanzó a cubrir los gastos aun dedicando todos sus ingresos a ello, por lo que se vieron obligados a incurrir en una descapitalización que redujo o agotó sus ahorros, a la venta de bienes o el endeudamiento. Suponemos, entonces, que si además hubieran tenido que efectuar el pago de la atención médica, el gasto habría sido insostenible para el hogar de la mayoría de los niños, en particular porque la población cubierta por el IMSS, y concretamente los casos estudiados, pertenecen a un estrato de población con ingresos mensuales relativamente bajos, menor de tres salarios mínimos $(70.6 \%)$.

Los motivos de gasto complementario referidos en las respuestas coinciden fundamentalmente con aquellos reportados en otros trabajos. El gasto en transporte, según lo reportado en la ENSAII, ${ }^{8}$ representa en promedio una tercera parte del costo de una consulta y la treceava parte del costo total de la atención. En nuestro estudio, puesto que se trata de población con acceso a la seguridad social, y que además es población que en algunos casos acude a centros de referencia desde otras zonas del país, los costos de transporte representan 30\% del costo total para los hogares. Los alimentos, conjuntamente con el transporte, constituyen los más frecuentes y con la mayor proporción de gastos complementarios en este tipo de evento.

La leucemia en menores de 15 años de edad, cuando es tratada adecuada y oportunamente, puede remitir clínicamente. Diversos estudios ${ }^{19}$ hechos en países como Estados Unidos de América, han señalado que en $80 \%$ de los casos los pacientes sobreviven los primeros cinco años posteriores a su diagnóstico. Para México, en un estudio realizado en la ciudad de Puebla, ${ }^{19}$ se informa una sobrevida de hasta cinco años en $85 \%$ de niños bien nutridos; este porcentaje es de $26 \%$ en los desnutridos. Si se considera que $40 \%$ de los casos incluidos en el estudio presentaban algún tipo de desnutrición, resulta entonces de especial importancia el seguimiento terapéutico de estos pacientes, al igual que su asistencia oportuna.

En los primeros dos años posteriores al diagnóstico se espera un promedio de seis periodos de hospitalización, antes de la estabilización de los pacientes, cada uno de ellos con duración diversa, así como en el número de visitas a control durante la remisión, proceso que, en promedio, se prolonga hasta tres años o más en algunos casos. Los resultados obtenidos en este estudio señalan que los desembolsos hechos por los hogares durante la primera hospitalización del enfermo con leucemia son demasiado elevados, por lo que es de suponer que los gastos subsiguientes, por la enfermedad sean insostenibles. Los gastos generados por las consultas y hospitalizaciones requeridas para que el padecimiento se controle o remita puede constituir una barrera para la continuidad del tratamiento. La ausencia de seguimiento terapéutico por insolvencia podría explicar el hecho de que esta enfermedad sea la primera causa de muerte por cáncer en pediatría en el país. ${ }^{10}$

\section{Conclusiones y recomendaciones}

Diversos estudios en el país muestran el incremento progresivo del gasto de bolsillo de los hogares dedicado a la atención médica. ${ }^{7}$ Hasta ahora la dinámica de dicho gasto se conoce parcialmente, y los análisis para medir la totalidad de los desembolsos que los hogares llevan a cabo para acceder a los centros de atención siguen siendo insuficientes.

Nuestros resultados muestran que para 14\% de los hogares entrevistados el costo durante la primera hospitalización de niños con leucemia representa un gasto catastrófico; asimismo, los hogares más afectados son los de menores ingresos, aquellos cuyo lugar de residencia es foráneo, y los que poseen un seguro de régimen no ordinario. Los hogares con menor ingreso son los que enfrentan el mayor impacto. Los gastos en transporte y alimentos resultaron ser los componentes más importantes del gasto. Si se toma en cuenta que en este estudio sólo se considera el costo de la primera hospitalización, y ésta se reconoce como uno de los eventos de mayor costo ${ }^{8,16}$ incluso en población asegurada, es posible que existan nuevas categorías de gasto que emerjan, posteriormente, al prolongar la atención. Por lo tanto, es de esperar que el impacto económico de la leucemia tenga consecuencias económicas aún mayores para estos hogares.

Futuros estudios pudieran analizar las diversas modalidades de costos indirectos, de costos y gastos en los hogares asociados con la evolución de la patología, ampliar el tiempo del estudio y medirlos en pacientes con diversos grados de avance de la enfermedad, cubrir a los hogares que no cuentan con seguridad social, comparar los costos en diferentes grupos de población e identificar aquéllos cuya necesidad de apoyo es imprescindible.

Los resultados sugieren la necesidad de evaluar la efectividad de las políticas de apoyo al derechoha- 
biente, ya que si bien la institución contribuye con una pequeña proporción para los gastos de traslado y pueden ocasionalmente concertar el funcionamiento de albergues para el alojamiento de familiares que no viven cerca de los centros de atención, dichas oportunidades no se conocen ni se utilizan, tampoco son diferenciadas por tipo de nivel económico ni por severidad del padecimiento. Se requiere operar una política de apoyo al paciente que proporcione información sobre los derechos con que cuentan para facilitar un acceso equitativo a los servicios.

Por otra parte, aunque existe una regionalización de los centros de atención de este padecimiento, que busca concentrar a los pacientes en diferentes áreas de servicio, el traslado de los pacientes fuera de su lugar de residencia sigue siendo necesario. Se requiere, por lo tanto, la evaluación económica de las alternativas posibles, incluyendo la creación de albergues regionalizados para alojar a los familiares y establecer en qué medida ello permitiría disminuir los costos de los hogares y facilitar la continuidad del tratamiento, mejorando con ello la sobrevida de estos pacientes. Las modalidades de los albergues ofrecidos pueden representar un bajo costo, relativamente, para la institución y constituir la única alternativa para la continuidad de la atención a grupos de bajos ingresos. Existen experiencias exitosas de su operación. El impacto económico de los costos complementarios de la atención se ha reconocido en otros países que cuentan con seguridad social. En Francia, las políticas de apoyo en el caso de patologías catastróficas incluen a los albergues como una prestación regular para quien los necesita. ${ }^{20}$

La magnitud de los efectos económicos de eventos inesperados, como la enfermedad, son grandes aun para aquellos hogares dentro del esquema de la seguridad social. Es de esperar que los gastos elevados imposibiliten la atención ante este tipo de eventos en los hogares de bajo ingreso que carecen de seguridad social. La implantación de las políticas de salud, orientadas a la provisión de un paquete de servicios mediante esquemas de aseguramiento financiados por medio del prepago que consideren los costos complementarios, puede ser una opción adecuada para proteger la economía de los hogares, sobre todo si se orienta hacia hogares de escasos recursos y aquéllos sin acceso a la seguridad social.

\section{Referencias}

1.Van Roijen L, Koopmanschap M, Rutten F,Van der M. Indirect cost of diseases: An international comparison. Health Policy 1995;33:15-29. 2. Gold M, Siegel J, Russell L,W einstein M. Cost-effectiveness in health and medicine. Massachusetts (MA): 0 xford University Press, 1996. 3. Avila L, G utiérrez C, Hernández P, Santos C, Silva L. EL costo social de la bronquitis crónica en la Ciudad de México: una experiencia piloto. Salud Publica Mex 1996;30(2):128-138.

4. D rummond M, Stoddart G, Torrance G. Métodos para la evaluación económica de los programas de atención a la salud. Madrid: Ed. Díaz de Santos, 1991.

5. Instituto Mexicano del Seguro Social. Ley del Instituto Mexicano del Seguro Social. México, D F: IMSS, 1998.

6. Instituto Mexicano del Seguro Social. Hacia el fortalecimiento y modernización de la seguridad social. México, DF: IMSS, 1996.

7. Secretaría de Salud. Programa N acional de Salud 2001-2006. México, DF: SSA, 2001.

8. Secretaría de Salud. Encuesta N acional de Salud II. México, DF: SSA, 1994.

9. Murray CJL, Knaul F, Musgrove P, Xu K, Kawabata K. D efining and measuring fairness in financial contribution to the health system. GPE. Ginebra:W HO, 2001; Discussion Paper Series. N 0. 24.

W HO .2001.D isponible en http://www3.who.int./whosis/ discussion_papers/pdf/paper24.pdf

10. Rivera-Luna R. Primer consenso de leucemia aguda linfoblástica pediátrica en México. Rev Invest Clin 1997;49:309-316.

11. Banco de México. Indicadores de precios.Tasa de inflación series 1997-2001. México, DF: Banco de México, 2002. Disponible en: http: ww.banxico.org.mx

12. Varian H. Microeconomía intermedia: un enfoque actual. 4ạ edición. España:Antoni Bosh, ed, 1996:163-202.

13. Grene W. Econometrics analysis. Fourth Edition. N ueva York (N Y): Prentice Hall,2000: 258.

14. Berrino F, Esteve J, Coleman MP. Basic issues in estimating and comparing the survival of cancer patients. Lyon: IARC Scientific Publications, 1995:1-14.

15. Poullier JP, Hernández P, Kawabata K. N acional health accounts: Sources, data and methods. G inebra:W H O ,2002; D iscussion Paper 47. W HO , 2002. Disponible En: http://www3.who.int./whosis/ discussion_papers/pdf/paper47.pdf

16.Yepes LFJ. Gasto privado en salud. Colombia 1977-1980. Gac Med Mex 1984;15(2):49-54.

17. Musgrove P. Family health care spending in Latin America. J Health Econ 1983;2:245-257.

18. Instituto Nacional de Estadística, G eografía e Informática. Encuesta $\mathrm{N}$ acional de Ingresos y $\mathrm{G}$ asto de los Hogares (EN IGH). México DF: IN EGI, 1996.

19. Lobato-Mendizábal E, Ruiz-Arguéllez G, Marín-López A. Leukemia ad nutrition. Malnutrition is an adverse prognostic factor in the outcome of treatment of patients with standard-risk acute lymphoblastic leukemia. Leuk Res 1989;13(10):899-906.

20. MISSO C. Social protection in the EU Member States and the European Economic Area. Luxemburgo: European Commission, 2000. 